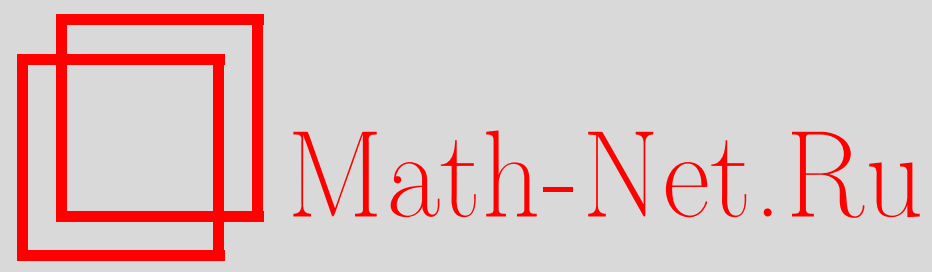

И. Х. Беккер, В. Н. Недов, О группах с изоморфными голоморфами, Матем. заметки, 1997, том 62, выпуск 3, 343-350

DOI: https://doi.org/10.4213/mzm1616

Использование Общероссийского математического портала Math-Net.Ru подразумевает, что вы прочитали и согласны с пользовательским соглашением http://www. mathnet.ru/rus/agreement

Параметры загрузки:

IP: 54.210 .77 .194

26 апреля 2023 г., 18:35:08

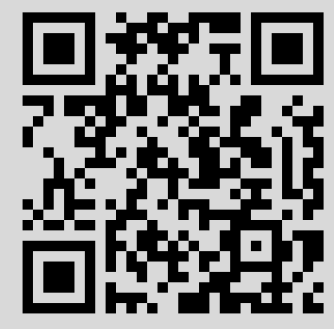




\title{
О ГРУППАХ С ИЗОМОРФНЫМИ ГОЛОМОРФАМИ
}

\author{
И. Х. Беккер, В.Н. Недов
}

Группы с изоморфными голоморфами назьваются голоморфно изоморфными. Рассматриваются с ледующие задачи.

1) Для каких абелевых групп голоморфный изоморфизм влечет изоморфизм самих групп?

2) Влечет ли голоморфный изоморфизм двух групп, одна из которых абелева, коммутативность другой группы?

Выделены классы групп, для которых даются положительные ответы на эти вопросы.

Библиограффия: 4 названия.

Группы с изоморфньми голоморфами назовем голоморфно изоморфным.ми. В работе изучаются свойства голоморфно изоморфных груп, соотношения между голоморфами абелевых групп и некоммутативных групп. Голоморфно изоморфные группы не всегда изоморфны. Известны примеры неизоморфных конечных некоммутативных голоморфно изоморфных групп. Например, при $n \geqslant 3$ группа диэдра и дициклическая группа порядка $4 n$ имеют изоморфные голоморфы [1]. Голоморфно изоморфные абелевы группы также не всег да изоморфны. Такой пример голоморфно изоморфных абелевых груш без кручения построен в п. 1. Однако можно выделить довольно широкие классы абелевых грушп, определяющихся своими голоморфами в категории $\bmod -Z$ всех абелевых групп [2, с. 148]. В п. 1 данной статьи доказано, что смешанная абелева $G$ група с нулевой 2-компонентой и периодической группой автоморфизмов Aut $G$ определяется своим голоморфом $\Gamma(G)$ в категории $\bmod -Z$ (теорема 1.1$)$.

В п. 2 работы рассматривается такой вопрос: влечет ли голоморфный изоморфизм групп $G$ и $G^{\prime}$, одна из которых абелева, коммутативность другой группы? Здесь выделены различные классы абелевых групा, для которых на этот вопрос дается положительньй ответ. Доказано, что если одна из двух голоморфно изоморфных групп является периодической без элементов порядка 2, то такие группы изоморфны (теоремы 2.1, 2.2).

Используемые в работе обозначения стандартны и соответствуют [3].

1. Голоморфно изоморфные абелевы группы. Отметим некоторые свойства голоморфов абелевых групा $G$, которые потребуются в дальнейшем. Пусть $\Gamma(G)$ - голоморф группы $G$, т.е. полупрямое расширение групы Aut $G$ с помощью групшы $G$. Всякий элемент группы $\Gamma(G)$ рассматриваем как пару $(g, \varphi), g \in G, \varphi \in$ Aut $G$. Групповая операция определена в $\Gamma(G)$ по правилу: если $(g, \varphi),(a, \psi) \in \Gamma(G)$, то $(g, \varphi)+(a, \psi)=$ $(g+\varphi a, \varphi \psi)($ или $(g, \varphi) \cdot(a, \psi)=(g \cdot \varphi a, \varphi \psi))$. Элементы грушшы $\Gamma(G)$ вида $(g, \varepsilon)$, где $\varepsilon-$ 
единица группы Aut $G$, образуют нормальную подгруппу, изоморфную группе $G$ (отождествляем ее с $G)$, а элементы вида $(0, \varphi)$, где 0 - нулевой элемент групшы $G$, образуют подгруппу, изоморфную Aut $G$ (отождествляем еe с Aut $G$ ). Итак, $\Gamma(G)=G \dashv \operatorname{Aut} G$, $G \cap \operatorname{Aut} G=\langle(0, \varepsilon)\rangle(\Gamma(G)=G \lambda$ Aut $G)$. Заметим, что $G$ является максимальной нормальной абелевой подгруппой групшы $\Gamma(G)$ и ее централизатор $C_{\Gamma(G)}(G)=G$. Пусть $H \triangleleft \Gamma(G), H_{1}, \Phi_{1}$ - соответственно множества всех первых, вторых компонент элементов подгруппы $H$. Тогда $H_{1}$ является характеристической подгруппой группы $G$, а $\Phi_{1}$ нормальной абелевой подгруппой групшы Aut $G$ [2, c. 139]. Нормальную подгрупшу $H$ голоморфа $\Gamma(G)$ групшы $G$ назовем голоморфно разложимой, если из того, что $(a, \varphi) \in H$ всегда следует $(a, \varepsilon),(0, \varphi) \in H$.

ЛЕмма 1.1 [2, с. 139]. Если $H$ - максимальная абелева нормальная подгруппа группь $\Gamma(G)$ и $H$ или $G$ не содержит әлементов порядка 2 , то $H$ - голоморфно разложсимая группа.

Если для любого автоморфизма $\theta \in \operatorname{Aut}(\Gamma(G))$ группа $G^{\theta}$ является голоморфно разложимой, то группу $G$ называем голоморфно разложимой. Согласно лемме 1.1 всякая абелева группа $G$ без элементов порядка 2 является голоморфно разложимой. Пусть $\Gamma(G)$ и $\Gamma\left(G^{\prime}\right)$ - соответственно голоморфы абелевых групп $G$ и $G^{\prime}, \mu: \Gamma(G) \longmapsto \Gamma\left(G^{\prime}\right)-$ изоморфное отображение. Характеристическую подгрупш $A$ групшы $G$ назовем $\mu$-голоморфно разложсимой, если $\mu A$ голоморфно разложима. В частности, групп $G$ назьвается $\mu$-голоморфно разложимой, если $\mu G$ голоморфно разложима.

ЛЕмма 1.2. Пусть $G$ и $G^{\prime}$ - голоморфно изоморфные абелевы группы, $\mu: \Gamma(G) \longmapsto$ $\Gamma\left(G^{\prime}\right) ; A, A^{\prime}$ - соответственно $\mu$-голоморфно, $\mu^{\prime}$-голоморфно разлохимие подәруппь әрупп $G, G^{\prime} ; A_{1}^{\prime}, \Phi_{1}^{\prime}$ и $A_{1}, \Phi_{1}$ - соответственно мнохсества всех первых, вторых компонент әлементов групп $\mu A$ u $\mu^{-1} A^{\prime}$.

Ecлu

$$
\mu A \cap G^{\prime} \leqslant A^{\prime} \quad u \quad \mu^{-1} A^{\prime} \cap G \leqslant A,
$$

mo

1) $A_{1}^{\prime}=\mu A_{1}, A=A_{1} \oplus A_{2}, A^{\prime}=A_{1}^{\prime} \oplus A_{2}^{\prime}$, где $A_{2}^{\prime}=\mu \Phi_{1}, A_{2}=\mu^{-1} \Phi_{1}^{\prime}$;

2) если всякий әлементарный автоморфизм группь $A$ (әруппь $A^{\prime}$ ) [4, с. 152] мохсно продолхить до некоторого автоморфизма группь $G$ (группь $G^{\prime}$ ), то $\Phi_{1}$ мономорфно вкладывается в $\operatorname{Hom}\left(A_{2}, A_{1}\right)$ ( $\Phi_{1}^{\prime}$ мономорфно вкладьвается в $\left.\operatorname{Hom}\left(A_{2}^{\prime}, A_{1}^{\prime}\right)\right)$.

ДокАЗАТЕЛЬСтво. Согласно условию имеем $\mu A=A_{1}^{\prime} \oplus \Phi_{1}^{\prime}, \mu^{-1} A^{\prime}=A_{1} \oplus \Phi_{1}$, притом $A_{1} \neq\langle(0, \varepsilon)\rangle, A_{1}^{\prime} \neq\left\langle\left(0, \varepsilon^{\prime}\right)\right\rangle$ - соответственно характеристические подгрупшы групп $G, G^{\prime}$ ( $\varepsilon^{\prime}$ - тождественный автоморфизм группы $\left.G^{\prime}\right)$.

1) Пусть подгруппы $A$ и $A^{\prime}$ удовлетворяют условию $(*)$. Имеем $A_{1}=\mu^{-1} A^{\prime} \cap G \leqslant A$ и $A_{1}^{\prime}=\mu A \cap G^{\prime} \leqslant A^{\prime}$. Отсюда следует $A_{1}=\mu^{-1} A^{\prime} \cap A$ и $A_{1}^{\prime}=\mu A \cap A^{\prime}$. Следовательно, $\mu A_{1}=A^{\prime} \cap \mu A=A_{1}^{\prime}$ и $A=A_{1} \oplus A_{2}, A^{\prime}=A_{1}^{\prime} \oplus A_{2}^{\prime}$, где $A_{2}=\mu^{-1} \Phi_{1}^{\prime}, A_{2}^{\prime}=\mu \Phi_{1}$.

2) Для любых $\varphi_{1} \in \Phi_{1}, \varphi_{1}^{\prime} \in \Phi_{1}^{\prime}, a_{2} \in A_{2}, a_{2}^{\prime} \in A_{2}^{\prime}$ имеем $\varphi_{1} a_{2}-a_{2}=\bar{a}_{1} \in A_{1}$, $\varphi_{1}^{\prime} a_{2}^{\prime}-a_{1}^{\prime}=a_{1}^{\prime} \in A_{1}^{\prime}\left[2\right.$, c. 139]. Тогда соответствие $a_{2} \mapsto \bar{a}_{1}$ определяет некоторьй гомоморфизм $\eta$ группы $A_{2}$ в $A_{1}$, т.е. $\varphi_{1}$ индуцирует гомоморфизм $\eta \in \operatorname{Hom}\left(A_{2}, A_{1}\right)$. Очевидно, отображение $\chi: \Phi_{1} \rightarrow \operatorname{Hom}\left(A_{2}, A_{1}\right), \chi \varphi_{1}=\eta$, есть мономорфизм. Аналогично получаем, что отображение $\chi^{\prime}: \Phi_{1}^{\prime} \rightarrow \operatorname{Hom}\left(A_{2}^{\prime}, A_{1}^{\prime}\right), \chi^{\prime} \varphi_{1}^{\prime}=\eta^{\prime} \in \operatorname{Hom}\left(A_{2}^{\prime}, A_{1}^{\prime}\right)$, есть также мономорфизм. 
СлЕДСТВИЕ 1.1. Пусть $\mu: \Gamma(G) \nrightarrow \Gamma\left(G^{\prime}\right), G_{1}^{\prime}, \Phi_{1}^{\prime} u G_{1}, \Phi_{1}$ - coomветственно мнохсества всех первых, вторых компонент әлементов групп $\mu G u \mu^{-1} G^{\prime}$. Если одна из әрупп $G, G^{\prime}$ является $\mu$-голоморфно разложимой (в частности, не содержит әлементов порядка 2), то

1) $G=G_{1} \oplus G_{2}, G^{\prime}=G_{1}^{\prime} \oplus G_{2}^{\prime}$, əде $G_{1}^{\prime}=\mu G_{1}, G_{2}^{\prime}=\mu \Phi_{1}, G_{2}=\mu^{-1} \Phi_{1}^{\prime}$;

2) $\Phi_{1} \cong \operatorname{Hom}\left(G_{2}, G_{1}\right), a \Phi_{1}^{\prime} \cong \operatorname{Hom}\left(G_{2}^{\prime}, G_{1}^{\prime}\right)$.

Пусть mod $-Z$ - категория всех абелевых груп. Говорим, что абелева группа $G$ определяется своим голоморфом $\Gamma(G)$ в категории $\bmod -Z$, если для любой группы $G^{\prime}$ из $\bmod -Z$ изоморфизм голоморфов $\Gamma(G)$ и $\Gamma\left(G^{\prime}\right)$ влечет изоморфизм групп $G$ и $G^{\prime}$.

Заметим, что группа $G$ не определяется своим голоморфом в категории $\bmod -Z$ тогда и только тогда, когда $\Gamma(G)$ содержит такую максимальную нормальную абелеву подгруппу $H$, что $\Gamma(G)$ и $\Gamma(H)$ изоморфны, но $G$ не изоморфна $H$.

Действительно, пусть групп $G$ не определяется своим голоморфом $\Gamma(G)$ в категории $\bmod -Z$ и $G, G^{\prime}$ такие голоморфно изоморфные группы, $G^{\prime} \in \bmod -Z$, что $G$ не изоморфна $G^{\prime}$ и $\mu: \Gamma(G) \longmapsto \Gamma\left(G^{\prime}\right)$. Тогда $\mu^{-1} G^{\prime}=H$ - максимальная нормальная абелева подгрупша групшы $\Gamma(G)$ не изоморфная $G$, а голоморфы $\Gamma(G)$ и $\Gamma(H)$ изоморфны.

Построим пример голоморфно изоморфных абелевых групп, не определяющихся своими голоморфами в категории $\bmod -Z$. Пусть $\mu_{1}: G \longmapsto \Gamma\left(G^{\prime}\right)$ и $\mu_{2}:$ Aut $G \longmapsto \Gamma\left(G^{\prime}\right)-$ такие мономорфизмы, что $\left\langle\mu_{1} g, \mu_{2} \varphi\right| g \in G, \varphi \in$ Aut $\left.G\right\rangle=\Gamma\left(G^{\prime}\right)$ и $\left\langle\mu_{1} g \mid g \in G\right\rangle \cap$ $\left\langle\mu_{2} \varphi \mid \varphi \in \operatorname{Aut} G\right\rangle=\left\langle\left(0, \varepsilon^{\prime}\right)\right\rangle$. Выясним, когда такая пара мономорфизмов $\left(\mu_{1}, \mu_{2}\right)$ индуцируется некоторым изоморфизмом $\mu: \Gamma(G) \longmapsto \Gamma\left(G^{\prime}\right)$ ? Ответ на этот вопрос дает

ЛЕмма 1.3. Пара мономорфизмов $\left(\mu_{1}, \mu_{2}\right)$ индуиируется некоторым изоморфизмом $\mu: \Gamma(G) \longmapsto \Gamma\left(G^{\prime}\right)$ тогда и только тогда, когда для любых $g \in G, \varphi \in$ Aut $G$

$$
\mu_{2} \varphi \cdot \mu_{1} g \cdot \mu_{2} \varphi^{-1}=\mu_{1} \varphi g
$$

Доказательство сводится к непосредственным вычислениям.

ПримеР 1.1. Пусть $G=A \oplus B \oplus\left(\bigoplus_{\mathfrak{N}} C\right), G^{\prime}=A \oplus B^{\prime} \oplus\left(\bigoplus_{\mathfrak{N}} C\right)$, где $A, B, C-$ группы без кручения ранга $1, p A \neq A$ для всякого простого числа $p$, тип $\tau(A)>\tau(B)$, $B^{\prime} \cong \operatorname{Hom}(B, A)$ и $\tau(C)$ не сравним с $\tau(A), \tau(B), \tau\left(B^{\prime}\right), \mathfrak{N}$ - произвольный кардинал. Имеем $\Gamma(G)=\Gamma(A \oplus B) \times \Gamma\left(\oplus_{\mathfrak{N}} C\right), \Gamma\left(G^{\prime}\right)=\Gamma\left(A \oplus B^{\prime}\right) \times \Gamma\left(\bigoplus_{\mathfrak{N}} C\right)$. Пользуясь леммой 1.3 , нетрудно проверить, что $\Gamma(A \oplus B) \cong \Gamma\left(A \oplus B^{\prime}\right)$. Отсюда следует, что голоморфы $\Gamma(G)$ и $\Gamma\left(G^{\prime}\right)$ изоморфны, но групшы $G$ и $G^{\prime}$ не изоморфны.

Теорема 1.1. Всякая $\mu$-голоморфно разложимая смешанная абелева группа $G$ (в частности, смешанная абелева группа $G$ без әлементов порядка 2) с периодической группой автоморфизмов Aut $G$ определяется своим голоморфом $Г(G)$ в категории $\bmod -Z$.

ДокАЗАТЕЛЬСТво. Пусть $G$ и $G^{\prime}$ голоморфно изоморфные абелевыгруппы и $G$ удовлетворяет условию теоремы, $\mu: \Gamma(G) \longmapsto \Gamma\left(G^{\prime}\right)$ и $\mu G \neq G^{\prime}$. Тогда согласно следствию $1.1 G=G_{1} \oplus G_{2}, G^{\prime}=G_{1}^{\prime} \oplus G_{2}^{\prime}$, где $G_{1}^{\prime}=\mu G_{1}, G_{2}^{\prime}=\mu \Phi_{1}, G_{2}=\mu^{-1} \Phi_{1}^{\prime}, G_{1}^{\prime}, \Phi_{1}^{\prime}$ и $G_{1}, \Phi_{1}$ - соответственно множества всех первых, вторых компонент элементов групп $\mu G$ и $\mu^{-1} G^{\prime}$, притом $\Phi_{1} \cong \operatorname{Hom}\left(G_{2}, G_{1}\right)$, а $\Phi_{1}^{\prime} \cong \operatorname{Hom}\left(G_{2}^{\prime}, G_{1}^{\prime}\right)$. Далее, $G_{1}, G_{1}^{\prime}$ - характеристические подгрупшы соответственно групп $G, G^{\prime}$ [2, с. 139]. Рассмотрим разложение $G=G_{1} \oplus G_{2}$. Так как Aut $G$ - группа периодическая, то $G$ - редуцированная группа. 
Пусть $\pi_{i}\left(t G_{i}\right)$ - множество простых чисел, к которому относится периодическая часть $t G_{i}$ группы $G_{i}(i=1,2)$. Покажем, что $t G \leqslant G_{1}$, т.е. $\pi_{2}\left(t G_{2}\right)=\varnothing$. Действительно, пусть $p \in \pi_{1}\left(t G_{1}\right) \cap \pi_{2}\left(t G_{2}\right)$. Тогда $G_{1}$ и $G_{2}$ имеют циклические прямые слагаемые порядка $p^{\alpha}$ для некоторых $\alpha \geqslant 1$ и $\operatorname{Hom}\left(G_{1}, G_{2}\right) \neq 0$. Это противоречит характеристичности $G_{1}$ в группе $G$. Допустим, что $\pi_{1}\left(t G_{1}\right) \cap \pi_{2}\left(t G_{2}\right)=\varnothing$ и $q \in \pi_{2}\left(t G_{2}\right)$. Тогда $G_{2}$ содержит примарную компоненту, каждый элемент которой неподвижен относительно всех автоморфизмов $\varphi_{1} \in \Phi_{1}$. Следовательно, $\mu^{-1} G^{\prime}=G_{1} \oplus \Phi_{1}$ не является максимальной нормальной абелевой подгруппой в $\Gamma(G)$, т.е. $C_{\Gamma(G)}\left(\mu^{-1} G^{\prime}\right) \neq \mu^{-1} G^{\prime}$, что невозможно. Доказано, что $t G \leqslant G_{1}$ и $\Phi_{1}, G_{2}$ - групшы без кручения. Если допустить, что $p G_{2} \neq G_{2}$ хотя бы для одного $p \in \pi_{1}\left(t G_{1}\right)$, то $\operatorname{Hom}\left(G_{2}, G_{1}\right)$ содержит гомоморфизмы порядка $p^{m}$ для некоторых $m \geqslant 1$. Такие гомоморфизмы получаем, строя отображения вида $G \rightarrow G / p^{k} G \rightarrow G_{1}=\left\langle g_{1}\right\rangle \oplus \bar{G}_{1}$, где $\left\langle g_{1}\right\rangle$ - циклическая подгруппа порядка $p^{\alpha}(\alpha \geqslant 1, k \geqslant 1)$. Получаем, что $\Phi_{1}$ содержит элементы конечного порядка, но $\Phi_{1}=\mu^{-1} G_{2}^{\prime}$, а $G_{2}^{\prime}$ - группа без кручения. В этом можно убедиться, рассматривая разложение $G^{\prime}=G_{1}^{\prime} \oplus G_{2}^{\prime}$. Если допустить, что $p G_{2}=G_{2}$ для некоторого $p \in \pi_{1}\left(t G_{1}\right)=\pi(t G)$, то $G_{2}$ имеет автоморфизмы бесконечного порядка. Следовательно, группа $G$ также имеет такие автоморфизмы, что противоречит условию. Теорема доказана.

2. Голоморфно абелевы группы. При изучении голоморфно изоморфных групп возникает такой вопрос: если одна из двух голоморфно изоморфных групп абелева, то будет ли другая группа абелевой?

ОПРЕДЕЛЕНИЕ 2.1. Абелеву группу $G$ назовем голоморфно абелевой, если из того, что $\Gamma(G) \cong \Gamma\left(G^{\prime}\right)$ для группы $G^{\prime}$ всякий раз следует, что $G^{\prime}$ - абелева групша.

Не всякая абелева группа является голоморфно абелевой. Однако, можно выделить довольно широкие классы голоморфно абелевых групा.

Лемма 2.1. Абелева группа $G$ не является голоморфно абелевой тогда и только тогда, когда ее голоморф $\Gamma(G)$ содержит такую неабелеву нормальную подzpynny $H$, чmo $\Gamma(G) \cong \Gamma(H)$.

ДокАЗАТЕЛЬСтво. Действительно, пусть $G$ не является голоморфно абелевой. Тогда существует такая неабелева группа $G^{\prime}$, что голоморфы групा $G$ и $G^{\prime}$ изоморфны, $\Gamma(G) \cong \Gamma\left(G^{\prime}\right)$. Пусть $\mu: \Gamma(G) \nrightarrow \Gamma\left(G^{\prime}\right)$ и $\mu^{-1} G^{\prime}=H$. Имеем $H \triangleleft \Gamma(G)$ и $\Gamma(G) \cong \Gamma(H)$.

Непосредственно проверяется

ЛЕмма 2.2. Пусть $H$ - нормальная подгруппа голоморфа $\Gamma(G)$ абелевой группь $G, H_{1}, \Phi_{1}$ - соответственно множсества всех первых, вторых компонент ее әлементов. Тогда

1) $H_{1}$ является характеристической подгруппой группь $G, a \Phi_{1}-$ нормальная nодгрynna гpynnbl Aut $G$;

2) если $(a, \varphi) \in H$, mo $(2 a, \varepsilon),\left(0, \varphi^{2}\right) \in H$;

3) если группа $G$ имеет автоморфизм $g \mapsto 2 g(g \in G)$, то $H$ - голоморфно разложимая подгруппа.

ЛЕмма 2.3. Всякая абелева группа $G$ с совершенным голоморфом $\Gamma(G)$ является голоморфно абелевой. 
ДокАЗАтЕЛЬСтво. Допустим противное: пусть голоморфы групш $G$ и $G^{\prime}$ изоморфны, $\Gamma(G) \cong \Gamma\left(G^{\prime}\right)$ и $G^{\prime}$ - группа неабелева. Но голоморф $\Gamma\left(G^{\prime}\right)$ имеет внешние автоморфизмы. Одним из них является автоморфизм вида $\omega:\left(a^{\prime}, \varphi^{\prime}\right) \mapsto\left(a^{\prime-1}, \psi_{a^{\prime}}^{-1} \varphi^{\prime}\right)$, где $\psi_{a^{\prime}} g^{\prime}=a^{\prime-1} g^{\prime} a^{\prime}, g^{\prime} \in G^{\prime}$, и $\omega\left(G^{\prime}\right)=C_{\Gamma\left(G^{\prime}\right)}\left(G^{\prime}\right) \neq G^{\prime}$.

СледСТВИЕ $2.1[2$, с. 146]. Абелева группа $G$ голоморфно абелева, если она удовлетворяет следующим условиям:

1) $G$ характеристична в своем голоморфе;

2) $G$ имеет автоморфизм $g \mapsto 2 g(g \in G)$.

Действительно, группа $G$, удовлетворяющая условиям 1) и 2), имеет совершенньй голоморф [2, с. 145].

Отметим еще несколько следствий леммы 2.3. При этом учтем теоремы 11.14-11.16 из $[2$, с. 146$]$.

СледствиЕ 2.2. 1) Абелева группа $G$ с автоморфизмом $g \mapsto 2 g(g \in G)$ голоморфно абелева, если Aut $G$ абелева.

2) Регулярно полная абелева группа $G$ без кручения голоморфно абелева, если Aut $G$ изоморфна одной из групп $Z(6), D C_{12}$ или $B T_{24}$ ( $G$ называется регулярно полной, если всякий ее автоморфизм $\varphi$, отличный от тождественного, регулярен, т.е. $\varphi g \neq g$ для $g \in G)$.

Если смешанная абелева группа $G$ имеет примарную компоненту $G_{p}=Z\left(p^{\infty}\right) \oplus$ $\left(\bigoplus_{i=1}^{k}\left\langle g_{i}\right\rangle\right)(1 \leqslant k<\infty)$, то $G=G_{p} \oplus G^{(p)}$. Пусть $\pi_{1}-$ множество простых чисел, к которому относятся ее примарные компоненты вида $G_{p}$.

СлЕдСТВИЕ 2.3. Смешанная нередуцированная 2-делимая абелева әруппа $G$ является голоморфно абелевой, если она удовлетворяет одному из условий:

1) $\pi_{1}=\varnothing$;

2) $\pi_{1} \neq \varnothing u p G^{(p)} \neq G^{(p)} \partial л я s \in \pi_{1}$.

Пусть $G$ - абелева группа без элементов порядка $2, G^{\prime}-$ неабелева и $\mu: \Gamma(G) \longmapsto \Gamma\left(G^{\prime}\right)$, $H=\mu^{-1} G^{\prime}, H^{\prime}=C_{\Gamma(G)}(H)=\mu^{-1} C_{\Gamma\left(G^{\prime}\right)}\left(G^{\prime}\right)$. Групша $\Gamma\left(G^{\prime}\right)$ имеет внешний автоморфизм $\omega$ вида $\omega(b, \psi)=\left(b^{-1}, \tau_{b}^{-1} \psi\right)$, где $\tau_{b}$ - внутренний автоморфизм группы $G^{\prime}$, $\tau_{b} g^{\prime}=b^{-1} g^{\prime} b\left(g^{\prime} \in G^{\prime}\right),(b, \psi) \in \Gamma\left(G^{\prime}\right)$.

Автоморфизм $\omega$ обладает такими свойствами:

1) отображает $G^{\prime}$ на ее централизатор $C_{\Gamma\left(G^{\prime}\right)}\left(G^{\prime}\right)$ относительно $\Gamma\left(G^{\prime}\right), G^{\prime \omega}=$ $C_{\Gamma\left(G^{\prime}\right)}\left(G^{\prime}\right) \neq G^{\prime}$

2) $\omega^{2}=\varepsilon$;

3) $\omega$ обращает элементы из центра $C\left(G^{\prime}\right)$ грушшы $G^{\prime}$.

Так как голоморфы $\Gamma(G)$ и $\Gamma\left(G^{\prime}\right)$ изоморфны, то автоморфизму $\omega$ соответствует некоторый внешний автоморфизм $\chi$ группы $\Gamma(G)$. Заметим, что $H^{\chi}=H^{\prime}$, а группы $H$ и $H^{\prime}$ голоморфно разложимы.

Действительно, пусть $(h, \varphi) \in H$, тогда $(2 h, \varepsilon),\left(0, \varphi^{2}\right) \in H$ (лемма 2.2$)$. Для любого $h^{\prime} \in H^{\prime}$ имеем $\left(h^{\prime}, \varepsilon\right)+(2 h, \varepsilon)+\left(-h^{\prime}, \varepsilon\right)=(2 h, \varepsilon)$, и так как группа $G$ не содержит элементов порядка 2 , то $h^{\prime}+h=h+h^{\prime}$. Следовательно, $h \in C_{\Gamma(G)}\left(H^{\prime}\right)=H$ и $H$ - голоморфно разложимая группа. Аналогично получаем, что группа $H^{\prime}$ также голоморфно разложима. 
Лемма 2.4. Пусть $G$ - абелева группа без элементов порядка 2. Тогда

а) $G^{\chi} \neq G u G^{\chi}=A \oplus \Phi$, где $A, \Phi$ - соответственно множества всех первы вторых компонент группь $G \chi$;

b) $A$ - характеристическая подгруппа группь $G$ и $A^{\chi}=A$;

c) $G=A \oplus B$, где $B=\Phi^{\chi^{-1}}$ u $B \cong \operatorname{Hom}(A, B)$.

ДокАЗАтЕЛьство. а) Допустим, что $G^{\chi}=G$. Рассмотрим нормальные подгруппы $H$ и $H^{\prime}$ голоморфа $\Gamma(G)$. Имеем $H=H_{1} \dashv \Phi_{1}, H^{\prime}=H_{1}^{\prime} \dashv \Phi_{1}^{\prime}$, где $H_{1}, H_{1}^{\prime}$ и $\Phi_{1}$, $\Phi_{1}^{\prime}$ - соответственно множества всех первых, вторых компонент элементов групп $H$ и $H^{\prime}$, притом $H_{1}$ и $H_{1}^{\prime}$ характеристичны в группе $G$, а $\Phi_{1}, \Phi_{1}^{\prime}$ - нормальные подгруппы группы Aut $G$. Так как $H_{1}^{\chi}=H_{1}$, то $2 H_{1} \leqslant H_{1} \leqslant H \cap H^{\prime}=C(H)$ - центр группы $H$. Тогда для любых $\varphi_{1} \in \Phi_{1}, h_{1} \in H_{1}$ получаем $(0, \varphi)+\left(2 h_{1}, \varepsilon\right)+\left(0, \varphi^{-1}\right)=\left(2 h_{1}, \varepsilon\right)$ или $\varphi\left(2 h_{1}\right)=2\left(\varphi h_{1}\right)=2 h_{1}$. Отсюда $\varphi h_{1}=h_{1}$ и $H$ - абелева группа, что невозможно. Следовательно, $G^{\chi} \neq G$, и по лемме 1.1 получаем $G^{\chi}=A \oplus \Phi$.

b) $A$ характеристична в группе $G\left[2\right.$, c. 140], $A=G^{\chi} \cap G$. Отсюда $A^{\chi}=\left(G^{\chi} \cap G\right)^{\chi}=$ $G \cap G^{\chi}$, так как $\chi^{2}=\varepsilon$.

c) Имеем $G^{\chi}=A \oplus \Phi$, отсюда $G=A^{\chi^{-1}} \oplus \Phi^{\chi^{-1}}=A \oplus \Phi^{\chi^{-1}}=A \oplus B$.

Всякий автоморфизм $\varphi \in \Phi$ индуцирует тождественньй автоморфизм на групе $A$ и для $b \in B$ имеем $(-b, \varepsilon)+(0, \varphi)+(b, \varepsilon)=(\varphi b-b, \varphi)$ и $\varphi b-b \in A$. Отсюда следует, что $\varphi$ определяет некоторьй гомоморфизм $\tau_{\varphi}: B \rightarrow A$. Нетрудно проверить, что $\Phi \cong \operatorname{Hom}(B, A)$. Следовательно, $B \cong \operatorname{Hom}(B, A)$.

СледСтвиЕ 2.4. Если $g=a+b \in G, a \in A, b \in B$, mо $\chi g=\left(a^{\prime}, \delta_{b}\right)$, әде $\delta_{b}-$ внутренний автоморфизм группы $\Gamma(G)$, индуиированный элементом $b \in B, a^{\prime} \in A$. Для любых $a \in A, b \in B, \chi a=a^{\prime} \in A, \chi b=\left(0, \delta_{b}\right), \chi\left(0, \delta_{b}\right)=b$.

Рассмотрим, далее, абелевы группы $G$ с несовершенными голоморфами $\Gamma(G)$.

ПРЕДЛОЖЕНИЕ 2.1. Абелева группа $G$ без әлементов порядка 2 с несовершенным голоморфом $\Gamma(G)$, характеристичная в своем голоморфе $\Gamma(G)$, является голоморфно абелевой әруппой.

ДоКАЗАТЕЛЬСТво. Пусть голоморф $\Gamma(G)$ изоморфен голоморфу $\Gamma\left(G^{\prime}\right)$ неабелевой группы $G^{\prime}, \mu: \Gamma(G) \longmapsto \Gamma\left(G^{\prime}\right), H=\mu^{-1} G^{\prime}, H^{\prime}=C_{\Gamma(G)}(H)$ - централизатор группы $H$ в $\Gamma(G)$. Имеем $H=H_{1} \dashv \Phi_{1}, H^{\prime}=H_{1}^{\prime} \dashv \Phi_{1}^{\prime}$, где $H_{1}, \Phi_{1}$ и $H_{1}^{\prime}, \Phi_{1}^{\prime}$ - соответственно подгруппы из всех первых, вторых компонент элементов групш $H$ и $H^{\prime}$. Так как $H$ и $H^{\prime}$ - нормальные подгрупшы групшы $\Gamma(G)$, то $H_{1}$ и $H_{1}^{\prime}$ характеристичны в $G$ (лемма 2.2 ) и в голоморфе $\Gamma(G)$. Группа $\Gamma(G)$ имеет внешний автоморфизм $\chi$, отображающий $H$ на $H^{\prime}, H^{\chi}=H^{\prime}$ и $H_{1}^{\chi}=H_{1}$. Следовательно, $H_{1}=H_{1}^{\prime}$. Если теперь учесть, что $H=C_{\Gamma(G)}\left(H^{\prime}\right)$, то получаем $\left.\Phi_{1}\right|_{H_{1}}=\langle\varepsilon\rangle, H$ - абелева группа, а $G$ - голоморфно абелева.

Заметим, что п. 1 следствия 2.2 вытекает также из предложения 2.1 .

СлЕДСТВИЕ 2.5. Каждая из следующих әрупп является голоморфно абелевой:

1) нередучированная группа без кручения;

2) әруппа без кручения с периодической группой автоморфизмов. 
Лемма 2.5. Пусть $\psi \in$ Aut $G$. Автоморфизм $\psi \in \Phi_{1}^{\prime}$ тогда и только тогда, $\kappa о г \partial а$

1) для любого $g \in G$ әлемент $\psi g-g \in H_{1}^{\prime}$;

2) $\left.\psi\right|_{H_{1}}=\varepsilon$.

ДоказАтельство. 1) Пусть $\psi \in \Phi_{1}^{\prime}, g \in G$. Имеем $(-g, \varepsilon)+(0, \psi)+(g, \varepsilon)=(\psi g-g, \psi)$. Отсюда $\psi g-g \in H_{1}^{\prime}$. Так как $\psi \in C_{\Gamma(G)}(H)=H^{\prime}$, то $\left.\psi\right|_{H_{1}}=\varepsilon$.

2) Обратно, пусть $\psi g-g \in H_{1}^{\prime}$ для любого $g \in G$ и $\left.\psi\right|_{H_{1}}=\varepsilon$. Для любого $\varphi \in \Phi_{1}$ имеем $\varphi g-g \in H_{1}, \psi(\varphi g-g)=\varphi g-g$ и $\varphi(\psi g-g)=\psi g-g$. Отсюда $\psi \varphi=\varphi \psi$. Следовательно, $\psi \in C_{\Gamma(G)}(H)=H^{\prime}$ и $\psi \in \Phi_{1}^{\prime}$.

ТЕорема 2.1. Всякая абелева периодическая группа $G$ с нулевой 2-компонентой является голоморфно абелевой.

ДоКАЗАТЕЛЬСТво. Пусть голоморф $\Gamma(G)$ группы $G$ изоморфен голоморфу $\Gamma\left(G^{\prime}\right)$ неабелевой групшы $G^{\prime}, \mu: \Gamma(G) \longmapsto \Gamma\left(G^{\prime}\right), H=\mu^{-1} G^{\prime}, H^{\prime}=C_{\Gamma(G)}(H)$. Согласно лемме 2.4 имеем $G=A \oplus B$, где $\operatorname{Hom}(A, B)=0, \operatorname{Hom}(B, A) \cong B$, подгруппы $H=H_{1} \dashv \Phi_{1}$, $H^{\prime}=H_{1}^{\prime} \dashv \Phi_{1}^{\prime}$ и $H_{1}, H_{1}^{\prime}$ - характеристические подгрупшы групшы $G$. Далее, $H_{1}=$ $\left(H_{1} \cap A\right) \oplus\left(H_{1} \cap B\right), H_{1}^{\prime}=\left(H_{1}^{\prime} \cap A\right) \oplus\left(H_{1}^{\prime} \cap B\right)\left[3\right.$, c. 60] и $H_{1} \cap B, H_{1}^{\prime} \cap B$ характеристичны в группе $B$, так как $H_{1}$ и $H_{1}^{\prime}$ характеристичны в $G$. Пусть $B=\bigoplus_{p \in \pi_{B}} B_{p}$, $B_{p}$ - примарная компонента группы $B, \pi_{B}$ - множество всех простых чисел, к которому относятся ее примарные компоненты. Очевидно, $H_{1} \cap B=\bigoplus_{p \in \pi_{1}}\left(H_{1} \cap B_{p}\right)$, $H_{1}^{\prime} \cap B=\bigoplus_{p \in \pi_{2}}\left(H_{1}^{\prime} \cap B_{p}\right)$, где $\pi_{1}, \pi_{2}$ - соответственно множества всех простых чисел, к которым относятся примарные компоненты групшы $H_{1} \cap B, H_{1}^{\prime} \cap B$.

Покажем теперь, что $B$ - группа конечная. Действительно, редуцированная периодическая групша $G$ с нулевой 2-компонентой голоморфно абелева [2, с. 146]. Поэтому далее $G$ - группа нередуцированная, $G=\bigoplus_{p \in \pi} G_{p}, A=\bigoplus_{p \in \pi_{A}} A_{p}$, где $G_{p}, A_{p}$ - соответственно примарные компоненты груп $G, A ; \pi, \pi_{A}$ - соответственно множества всех простых чисел, к которому относятся их примарные компоненты. Имеем $\pi_{B} \subseteq \pi_{A}$. Допустим, что $p \in \pi_{B}$, но $p \notin \pi_{A}$. Тогда, рассмотрев группу $\operatorname{Hom}(B, A) \cong \operatorname{Hom}\left(B_{p}, A\right) \oplus$ $\operatorname{Hom}\left(\bigoplus_{q \neq p} B_{q}, A\right)$, заключаем, что $\operatorname{Hom}\left(B_{p}, A\right)=0$ и $p$-компонента группы $\operatorname{Hom}(B, A)$ также нулевая. Следовательно, групп $\operatorname{Hom}(B, A)$ не изоморфна $B$, что невозможно. Заметим, что всякая группа $A_{p}$ является делимой группой. В противном случае группа $G$ имеет автоморфизмы $\omega$, для которых $A^{\omega} \neq A$, что противоречит характеристичности $A$ в группе $G$. Всякая же группа $B_{p}$ является редуцированной. Если допустить противное, то $\operatorname{Hom}(B, A)$ имеет хотя бы одно прямое слагаемое, изоморфное $\operatorname{Hom}\left(Z\left(p^{\infty}\right), Z\left(p^{\infty}\right)\right)$, что противоречит периодичности $G$. Если допустить, что хотя бы одна групша $B_{p}$ является бесконечной, то получаем, что $\operatorname{Hom}(B, A)$ содержит элементы бесконечного порядка [3, теорема 47.1, с. 233]. Следовательно, всякая група $B_{p}$ - группа конечная. Так как $B \cong \operatorname{Hom}(B, A) \cong \prod_{p \in \pi_{B}} \operatorname{Hom}\left(B_{p}, A\right)$, то $\pi_{B}-$ множество конечное. Отсюда следует, что $B$ - группа конечная.

Убедимся, что $\pi_{1} \cap \pi_{2}=\varnothing$. Для этого достаточно показать, что $\left(H_{1} \cap B\right) \cap\left(H_{1}^{\prime} \cap B\right)=0$. Допустим противное, пусть $0 \neq b \in\left(H_{1} \cap B\right) \cap\left(H_{1}^{\prime} \cap B\right)$. Тогда при действии автоморфизма $\chi$ получаем $\chi b=\left(0, \delta_{b}\right)$, где

$$
\delta_{b} \in\left(\begin{array}{cc}
\varepsilon & \operatorname{Hom}(B, A) \\
0 & \varepsilon
\end{array}\right) .
$$


В то же время $b \in C(H)=H \cap H^{\prime}$ и $\chi b \neq\left(0, \delta_{b}\right)$. Противоречие. Следовательно, $\pi_{1} \cap \pi_{2}=\varnothing$. Очевидно, $B_{p} \cong \operatorname{Hom}\left(B_{p}, A\right)$ и для $c \in B_{p}, q \neq p\left(p, q \in \pi_{B}\right)$ имеем $\left.\delta_{c}\right|_{A}=\varepsilon,\left.\delta_{c}\right|_{B_{q}}=\varepsilon, \delta_{c} b^{\prime}=b^{\prime}+\psi b^{\prime}$, где $b^{\prime} \in B_{p}, \psi \in \operatorname{Hom}\left(B_{p}, A\right)$. Пусть теперь $p \in \pi_{2}$, тогда автоморфизм $\delta_{c}$ действует тождественно на группе $H_{1}$ и для любого $g \in G$ имеем $\delta_{c} g-g \in H_{1}^{\prime}$. По лемме 2.5 получаем $\delta_{c} \in \Phi_{1}^{\prime}$ и так как $\left(H^{\prime}\right)^{\chi^{-1}}=H=\left(\left(H_{1} \cap A\right) \oplus\right.$ $\left.\left(H_{1} \cap B\right)\right) \dashv \Phi_{1}$, то $\chi^{-1} \delta_{c}=b^{\prime} \in H_{1} \cap B$. Это противоречит тому, что $\pi_{1} \cap \pi_{2}=\varnothing$. Следовательно, $G^{\prime}$ - групша абелева. Теорема доказана.

ЗАмЕчАниЕ. Всякая периодическая абелева группа $G$ с нулевой 2-компонентой определяется своим голоморфом в категории $\bmod -Z[2$, с. 150$]$.

Следовательно, имеет место такая

ТЕОРема 2.2. Если одна из двух голоморфно изоморфных групп $G$ и $G^{\prime}$ является абелевой периодической с нулевой 2-компонентой, то такие группы изоморфны.

\section{СПИСОК ЦИТИРОВАННОЙ ЛИТЕРАТУРЫ}

[1] Mills W. H. Multiple holomorphs of finitely generated abelian groups // Trans. Amer. Math. Soc. 1951. V. 71. № 3. P. 379-392.

[2] Беккер И.Х., Кожухов С.Ф. Автоморфизмы абелевых групп без кручения. Томск: Изд-во Томского ун-та, 1988.

[3] Фукс Л. Бесконечные абелевы группы. Т. 1. М.: Мир, 1974.

[4] Басс Х. Алгебраическая $K$-теория. М.: Мир, 1973.

Томский государственный университет 